\title{
Nevoid hyperkeratosis of the nipple and areola
}

\section{Abbasov Aykhan', Özçınar Beyza²}

\author{
${ }^{1}$ Department of General Surgery, Liv Hospital Ulus, Istanbul, Turkey, ${ }^{2}$ Department of General Surgery, Istanbul Faculty of \\ Medicine, Istanbul University, Istanbul, Turkey
}

Corresponding author: Abbasov Aykhan, MD, E-mail: dr.ayxan.abbasov@gmail.com

\begin{abstract}
Nevoid hyperkeratosis of the nipple and areola is an extremely rare and benign skin disease of unknown etiology, predominantly seen in childbearing aging women, especially in the second and third decades of life. The disease is characterized by gradually growing verrucous thickening and brown pigmentation of the nipple. Here, we present a case of nevoid hyperkeratosis of the nipple and areola in a 42-year-old female patient. Although nevoid hyperkeratosis of the nipple and areola has a benign character, it is a problem for patients due to deformity and for clinicians because of its similarity to paget disease. There is no consensus on treatment; Topical agents such as keratolytics, steroids, retinoids or calcipotriol, and ablative methods such as cryotherapy, carbon dioxide laser, radiofrequency, or shaving excision are treatment options.
\end{abstract}

Key words: Nevoid hyperkeratosis; nipple; areola

\section{INTRODUCTION}

Hyperkeratosis of the nipple and areola is an extremely rare condition $[1,2]$. Nevoid hyperkeratosis is a benign disease characterized by pigmentation and thickening of nipple and areola, which may involve one or both breasts [1]. Nevoid hyperkeratosis of the nipple and areola (NHNA) is often seen in women of childbearing age, especially in the 2 nd and 3rd decade. Its increase during pregnancy suggests the hormonal relation [3]. However, the underlying etiology is not fully understood.

NHNA constitutes a serious aesthetic and psychological problem especially in bilateral and widespread involvement of both nipple and areola. Here, we report a case of nevoid hyperkeratosis of the nipple and areola.

\section{CASE REPORT}

A 42-year-old woman presented to our breast clinic with a complaint of black-colored, irregular protuberances/ thickenings on the left breast covering the whole nipple and areola complexes, which have existed since her puberty. Her medical history revealed 2 vaginal deliveries and one miscarriage. She was no hormone usage and good in general health. There was no similar disease or any breast cancer case in her family. The patient mentioned that the lesions in the nipple and areola became larger during pregnancies. Her physical examination revealed diffuse heterogenous hyperpigmentation, plague-like thickening of the left nipple, and areola complexes with papillomatous surfaces (Figs. la and lb). There was no mass lesion and axillary lymphadenopathy detected in both breasts and axilla. The bilateral mammography and ultrasound scans were normal. There were no nevoid lesions or keratosis or any dermatological lesions in her whole body other than left breast nipple-areola complex. Dermatological consultation revealed

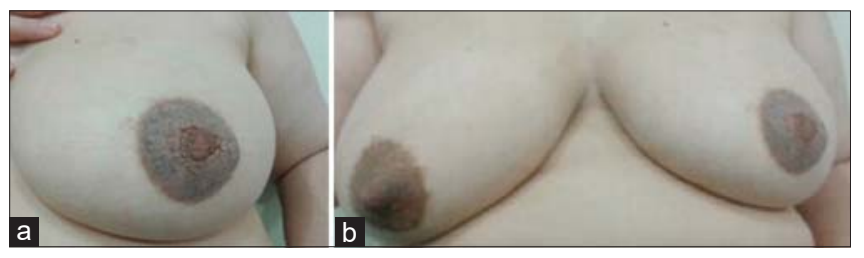

Figure 1: $(a$ and $b)$ Diffuse heterogenous hyperpigmentation, plaguelike thickening of left nipple and areola complexes with papillomatous surfaces in the nipple and areola.

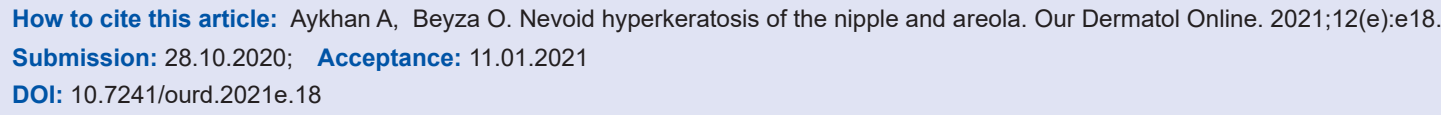


nevoid hyperkeratosis of the nipple and areola in the left breast. No medical or surgical treatment was recommended.

\section{DISCUSSION}

NHNA was first described in 1923 as a benign skin disease [3]. NHNA can occur in both sexes, and 80\% of cases reported to date are women [1-3]. Although the etiology is not known exactly, there are histories of estrogen usage or spironolactone or anticancer drug usage in some cases [4]. However, in our case, there was no history of any medication.

There are two types of hyperkeratosis of nipple and areola. Primary or idiopathic and secondary. If it is accompanied by other skin lesions such as epidermal nevus, seborrheic keratosis, acanthosis nigricans, or the lesions that develop after drug use are called secondary type [5]. In our patients, there was no other skin lesions or medicine usage and her lesions are idiopathic. In differential diagnosis, seborrheic keratosis, hyperkeratosis due to itching, mycosis fungoides, and pigmented basal cell carcinoma are included [6].

In NHNA, patients are usually asymptomatic, but the lesions do not regress without treatment. Keratolytic topical agents should be preferred in the first-line treatment. If there is itching, topical steroids can be added to treatment [2]. Also, topical retinoids and vitamin $\mathrm{D}$ analogs are the other treatment options. Surgical excision can be recommended in patients who are cosmetically and psychologically uncomfortable. Complete excision of the nipple/ areola complex and reconstruction after excision or shaving of the nipple and areola are surgical treatment alternatives. Therefore, radio frequency ablation or cryotherapy, or carbon dioxide laser is the other treatment options. In our patient, she did not want any treatment, and only follow up was recommended to the patient.

\section{CONCLUSION}

Nevoid keratosis is one of the rare causes of epidermal nevus. Although it has a benign character, it is a problem for patients due to deformity and for clinicians because of its similarity to paget disease. There is no consensus on treatment; Topical agents such as keratolytics, steroids, retinoids or calcipotriol, and ablative methods such as cryotherapy, carbon dioxide laser, radiofrequency, or shaving excision are treatment options.

\section{Consent}

The examination of the patient was conducted according to the principles of the Declaration of Helsinki.

The authors certify that they have obtained all appropriate patient consent forms, in which the patients gave their consent for images and other clinical information to be included in the journal. The patients understand that their names and initials will not be published and due effort will be made to conceal their identity, but that anonymity cannot be guaranteed.

\section{REFERENCES}

1. Hariharasubramony A, Chankramath $\mathrm{S}$. Hyperkeratosis of nipple and areola. Our Dermatol Online. 2012;3:215-6.

2. Ghanadan A., Balighi K., Khezri S., Kamyabhesari K. Nevoid hyperkeratosis of the nipple and/or areola: treatment with topical steroid. Indian J Dermatol. 2013;58:408.

3. Higgins HW, Jenkins J, Horn TD, Kroumpouzos G. Pregnancyassociated hyperkeratosis of the nipple. JAMA Dermatol. 2013;149:722.

4. Carr ES, Brown SC, Fiala KH. Painful nipple hyperkeratosis secondary to vemurafenib. Dermatol Ther. 2017;30(3) doi: 10.1111/ dth.12477.

5. Mahjoub T. Unilateral nevoid hyperkeratosis of the nipple and areola in a Saudi female. Our Dermatol Online. 2018;9:312-5.

6. Raffas W, El Amrani F, Benzekri L, Benzekri A, Senouci K, Hassam B. Successful treatment with calcipotriol for nevoid hyperkeratosis of the nipples. Clin Med Insights Dermatol. 2013;6:27-30.

Copyright by Abbasov Aykhan, et al. This is an open-access article distributed under the terms of the Creative Commons Attribution License, which permits unrestricted use, distribution, and reproduction in any medium, provided the original author and source are credited.

Source of Support: There is no financial support. Conflict of Interest: Authors have not conflicts of interest. 